\title{
Spore Swelling and Germination in Fusarium culmorum
}

\author{
By R. MARCHANT AND MONICA F. WHITE \\ Department of Botany, University College, Gower Street, \\ London, W.C. 1
}

(Received 24, June 1965)

\section{SUMMARY}

Carbon and nitrogen sources were found to be necessary both for the germination and the associated swelling of the macroconidia of Fusarium culmorum. The swelling of the spores took place before the emergence of the germ tube and was measured by two methods. The spores were shown to have a mucilaginous covering, which affected the uptake of nutrients and into which glucose was absorbed by a physical process rather than by an enzymic one. Tritiated water was used to demonstrate that the swelling of the spores in the presence of a nitrogen source was due largely to water uptake and only in a small part to an increase in dry weight.

\section{INTRODUCTION}

The germination of fungal spores has received a great deal of attention but in general the available data cover many species with few examples of detailed studies. Activation and germination of Neurospora tetrasperma ascospores (Goddard, 1935; Sussman, 1954) and germination of basidiospores of Schizophyllum commune (Hafiz \& Niederpruem, 1963; Ratts, Hafiz, Niederpruem \& Egbert, 1964) have both received extensive investigation.

Fusarium culmorum exists parasitically and saprophytically on cereal crops, producing asexual fusiform to falcate macroconidia only, which are 3- to 5-septate and 25-50 $\mu$ long by 5-7 $\mu$ wide. The mycelial and conidial chlamydospores which are produced by this fungus were not investigated in the present study. The macroconidia germinate in liquid culture in $4-6 \mathrm{hr}$, after their swelling. Ekundayo \& Carlile (1964) studied the similar swelling which occurs before the germination of sporangiospores of Rhizopus arrhizus. They postulated a system of active water uptake and a permeability change in the cell wall, although they did not investigate relative changes in dry weight and water content of the spores. The present study was designed to investigate the swelling of the macroconidia of $F$. culmorum and the relative contributions of water uptake and dry weight changes to this swelling.

\section{METHODS}

Organism. The strain of Fusarium culmorum used was obtained from the Commonwealth Mycological Institute (IMI. 96283) and was a typical isolate in all respects. The organism was grown on potato sucrose agar in large culture bottles at $25^{\circ}$ in an incubator which had glass doors and was exposed to normal daylight.

Culture medium. The standard culture medium was potato sucrose agar, containing the filtered extract of $250 \mathrm{~g}$. potato in 11 . medium, supplemented with $2 \%$ 
$(w / v)$ sucrose. The culture bottles were normally inoculated with a conidial suspension in sterile water.

Preparation of spore suspensions. The conidial suspensions used in the experiments were prepared by washing conidia from the surface of a culture with a small quantity of sterile distilled water. Mycelial fragments were removed by filtration through glass wool and the conidia then washed three times with water. Conidial suspensions for use were adjusted to a turbidity reading of 2.0 at $610 \mathrm{~m} \mu$ on a Hilger colorimeter, which represented a dry weight of about $2 \mathrm{mg} \cdot / \mathrm{ml}$. Washing the conidia was shown to have no effect on their germinative capacity, in agreement with the results of Cochrane, Cochrane, Simon \& Spaeth (1963).

The assessment of germination. Germination experiments were done at $25^{\circ}$ in media buffered with $0.03 \mathrm{M}$-phosphate buffer ( $\mathrm{pH} 6.5)$ and germination was recorded as having occurred when a visible germ tube had been produced. Germination counts were made after shaking for $6 \mathrm{hr}$. Optical measurements of maximum conidial width were made with a micrometer eyepiece. The media for conidial swelling and dry-weight determinations contained $50 \mu$ moles glucose $/ \mathrm{ml}$. and $20 \mu$ moles ammonium sulphate/ml. in $0.03 \mathrm{M}$-phosphate buffer (pH 6.5).

Dry-weight determinations. Measurements of dry weight were done in glass tubes which had been previously dried and weighed. Samples of conidia suspended in medium were placed in these tubes and centrifuged. The supernatant fluid was discarded and the tube containing the pellet of conidia was dried to a constant weight at $80^{\circ}$. In experiments in which the conidia were washed after sampling the pellet was resuspended in $5 \mathrm{ml}$. distilled water and recentrifuged before drying.

Manometric methods. Oxygen uptake was measured in the usual Warburg apparatus at $25^{\circ}$, with direct absorption of carbon dioxide by $10 \%$ (w/v) $\mathrm{KOH}$.

Tritium labelling. Estimations of relative water uptake were made after $4.5 \mathrm{hr}$ at $25^{\circ}$, before germ-tube emergence, by using tritiated water. The conidia were spun down from the tritium-labelled medium containing $1 \mathrm{mC}$. tritium, and were resuspended in $1 \mathrm{ml}$. of unlabelled water. Samples $(0.01 \mathrm{ml}$.) of the resuspended conidia were counted in a liquid scintillation counter, with an NE 220 scintillator liquid.

Mucilage staining. The mucilaginous covering of the macroconidia was revealed by staining with $0.25 \%$ aqueous thionine for $10-15 \mathrm{~min}$., after fixation in acetic acid + ethanol $(1+3)$ saturated with mercuric chloride. The conidia were fixed to a slide with Haupt's adhesive and mounted in $10 \%$ aqueous nigrosin. The mucilaginous coat was then visible as a thin faintly stained layer.

Inhibitor methods. Cyanide inhibition was achieved by supplying $10^{-2} \mathrm{M}-\mathrm{KCN}$ in shake cultures.

\section{RESULTS}

\section{Requirements for spore germination}

Cochrane, J. C. et al. (1963) found that macroconidia of Fusarium solani f. phaseoli required an exogenous supply of carbon and nitrogen sources for germination, and also a factor present in yeast extract which could be completely replaced by ethanol or acetoin and partially replaced by acetaldehyde or several amino acids. Sisler \& Cox (1954) showed that washed conidia of Fusarium roseum required only carbon and nitrogen sources for germination. The requirements of $F$. culmorum 
macroconidia are similar to those reported by Sisler \& Cox, as can be seen from Table 1 . The conidia were capable of about $14 \%$ germination in distilled water, but the maximum values were only achieved in the presence of carbon + nitrogen sources. Ammonium sulphate was the most efficient source of nitrogen in comparison with potassium nitrate and potassium nitrite; asparagine was capable of serving as carbon + nitrogen source. Various sugars, including fructose, xylose, arabinose, galactose, glucose and lactose, were tested as carbon sources; all supported germination and were utilized to some extent (Fig. 1). Acetate did not support germination, although measurements of oxygen uptake showed that it was utilized. This result is in agreement with Cochrane, Cochrane, Vogel \& Coles (1963), who found that acetate did not support germination in $F$. solani f. phaseoli without the addition of the yeast extract factor.

\section{Table 1. Percentage germination of macroconidia of Fusarium culmorum in various media at $25^{\circ}$}

Germination in distilled water varied between 11 and $14 \%$ in different experiments and therefore the results were standardized by expressing germinations as percentages above the value in distilled water.

\section{Medium \\ 0.03 M-phosphate buffer ( $\mathrm{pH} \mathrm{6.5)}$ \\ 0.01 m-glucose \\ 0.008 M-ammonium sulphate \\ $0.008 \mathrm{M}$-ammonium sulphate $+0.01 \mathrm{M}$-glucose \\ 0.004 M-potassium nitrate +0.01 M-glucose \\ Oxygen uptake during germination}
$\%$ germination
above the
value in
distilled water

$27 \cdot 8$

Oxygen uptake by germinating conidia was followed in media containing carbon and nitrogen sources. Carbon was supplied as D-glucose (10 $\mu$ moles/flask) and nitrogen as ammonium sulphate (25 $\mu$ moles/flask) in media buffered at $\mathrm{pH} 6.5$ with $0.03 \mathrm{M}$ phosphate buffer. The graphs for oxygen uptake are given in Fig. 2. The graphs for glucose utilization show an initial period of about $1 \mathrm{hr}$ when the rate of oxygen uptake gradually increased until linearity was reached. This period is unlikely to represent an enzyme induction, partly because of the short time involved and partly because the organism had been grown on a medium containing glucose. The delay in reaching maximum oxygen uptake would thus appear to be due to some barrier, physical or metabolic, to the entry of glucose into the cells.

When a limited amount of glucose was supplied to the spores it was exhausted at about the same time in the presence or absence of ammonium sulphate, although more was oxidized in the presence of the nitrogen source. Ammonium sulphate supplied in the absence of a carbon source had no effect on the basal respiration rate. The ammonium sulphate must have a profound effect on the fate of carbon in the germinating spore, as is borne out by the failure to germinate in its absence.

\section{Conidial swelling}

When conidia of Fusarium culmorum were germinated in a medium containing glucose and a nitrogen source the majority showed a marked swelling, leading to 
distension of the wall between the septa (Plate). Conidia germinated in a medium lacking nitrogen showed no such swelling. The swelling of the conidia was measured directly during the period preceding germ-tube emergence by optically following the maximum conidial width. The results in terms of $\%$ increase in mean maximum conidial width for samples of 50 conidia are shown in Fig. 3 together with the standard errors of the points. It can be seen that, while the conidia in glucose + ammonium sulphate showed a near-linear increase in width, those in glucose alone did not significantly differ from the initial sample or from the buffer control.

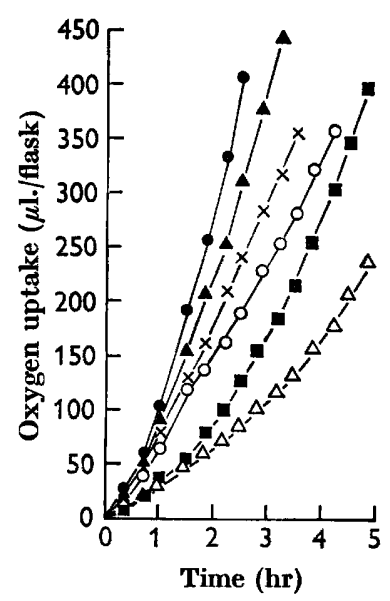

Fig. 1

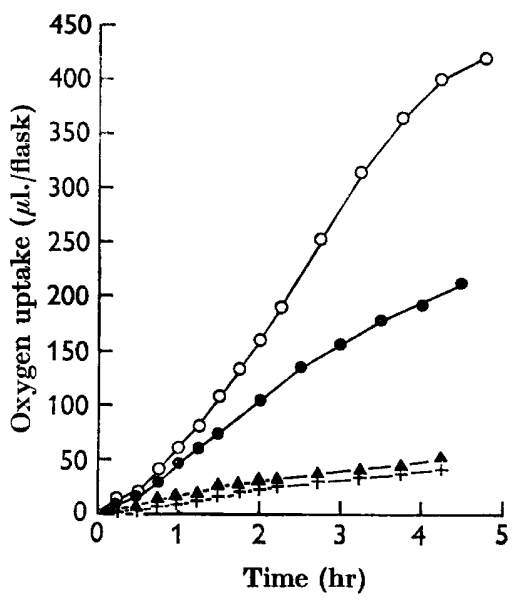

Fig. 2

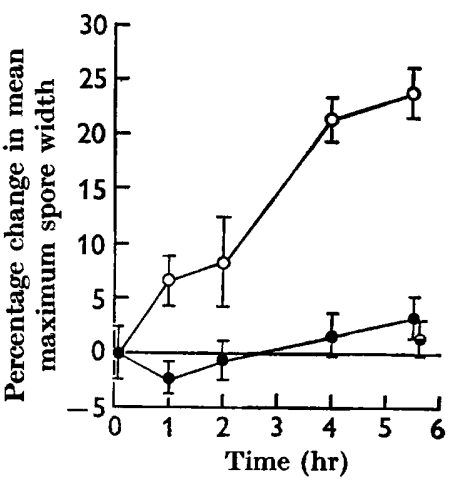

Fig. 3

Fig. 1. Utilization of various sugars by germinating macroconidia of Fusarium culmorum, as measured by oxygen uptake at $25^{\circ} . \triangle-\Delta$, Lactose; $\square-\square$, arabinose; $O-O$, xylose; $\times-\times$, galactose; $\Delta-\Delta$, fructose; -0 , glucose.

Fig. 2. The oxidation of glucose $(10 \mu$ moles $)$ in the presence or absence of ammonium sulphate, and the effect of ammonium sulphate on the basal respiration rate of, macroconidia of Fusarium culmorum. - - , Glucose; $O-O$, glucose + ammonium sulphate; +-+ , ammonium sulphate; $\Delta-\Delta$, control. Oxygen uptake was measured at $25^{\circ}$.

Fig. 3. Percentage change in mean maximum conidial width for macroconidia of Fusarium culmorum shaken in: - - , glucose; $\bigcirc-\bigcirc$, glucose + ammonium sulphate; $\ominus$, buffer (pH.) The optical measurements were made on samples of 50 conidia and the standard errors are shown. The experiment was done at $25^{\circ}$.

As a complementary method a precipitation technique was devised to estimate total changes in conidial volume. The method depends on the fact that the conidia of Fusarium culmorum precipitate easily and because of their shape pack down in a regular layered fashion. Samples of conidia in liquid medium were put into sealed lengths of $2 \mathrm{~mm}$. bore glass tubing. The conidia were then centrifuged for $2 \mathrm{~min}$. at constant speed (1400g) and the depth of the precipitate expressed as a proportion of the total height of the column of liquid. The method was applied to the same set of conditions that had been examined by the optical method. The $\%$ changes in the volume parameter are expressed graphically in Fig. 4. From the results obtained with the optical measurement method it was to be expected that any increase would be linear, but in the presence of glucose, whether ammonium sulphate was present or not, there was a sudden rise during the first hour followed by the predicted linear 
increase. The rate of increase over the linear portion of the graph agrees well with the rate found by optical measurement for conidia in glucose + ammonium sulphate. The buffer control showed no rapid initial increase. The similarity of time scales suggested that increasing oxygen uptake and overall conidial swelling might be linked phenomena. As optical measurement does not reveal the initial increase it is implied that the cause of this swelling was a layer outside the conidial wall which was not visible in unstained preparations. The nature of this layer is suggested by the work of Drs J. M. Hirst and H. L. Nixon (personal communication 1965), who



Fig. 4

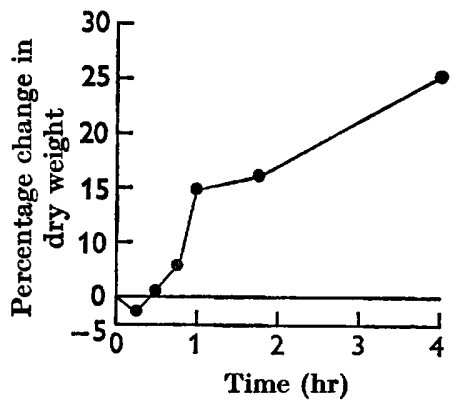

Fig. 5

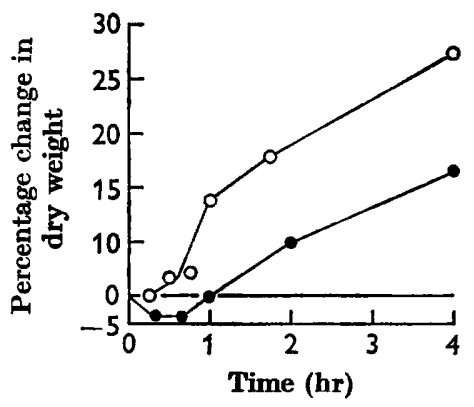

Fig. 6

Fig. 4. Percentage change in conidial size, as measured by the precipitation method, for macroconidia of Fusarium culmorum shaken in: -0 , glucose; $\mathrm{O}-\mathrm{O}$, glucose + ammonium sulphate; $\ominus$, buffer (pH 6.5). The experiment was done at $25^{\circ}$.

Fig. 5. Percentage change in dry weight for macroconidia of Fusarium culmorum shaken in glucose medium at $\mathbf{2 5}^{\circ}$ and unwashed before drying.

Fig. 6. Percentage change in dry weight for macroconidia of Fusarium culmorum shaken in glucose +ammonium sulphate at 25: $\mathrm{O}-\mathrm{O}$, unwashed before drying; -0 , washed before drying.

showed a mucilaginous covering on the wettable microconidia of Fusarium oxysporum by an electron microscope replica technique. The conidia of $F$. culmorum were examined for any mucilaginous layer by a staining technique with thionine and nigrosin. This showed that conidia shaken in any medium containing glucose had a distinct faintly-staining layer, 0.5-1 $\mu$ thick, which surrounded the conidia. This layer was not detectable on conidia freshly sampled or on conidia shaken in buffer. Although this layer did not show the normal intense staining reaction of mucilage (Wilson, 1965), it is obviously responsible for the rapid overall increase in size of the conidia in glucose-containing media.

\section{Dry-weight changes}

Associated with the respiration of glucose is its oxidative assimilation, with a consequent increase in dry weight. Experiments were therefore designed to investigate the changes in dry weight of conidia during germination, especially during the first hour, and the role of the mucilaginous sheath in nutrient uptake. The increase in dry weight was determined in conidial samples shaken in the presence of glucose and glucose + ammonium sulphate, without washing the samples before 
drying. The results are shown in Figs. 5 and 6; once again there was the rapid increase over the first hour, followed by a period of linear increase up to the time of germination, when the increase became exponential. To determine whether the uptake of glucose into the mucilaginous sheath was a physical process or an enzymic one, samples of conidia were washed before drying; the results of this experiment are shown in Fig. 6. If the binding of glucose into the sheath were enzymic, washing ought not to remove it; but the graph shows that there was no increase in dry weight over the first hour, indicating that the glucose in the sheath had been removed. The dry weight does show the normal linear increase after the first hour. The observation that various sugars, pentoses and hexoses, were taken through the mucilage and reached a maximum rate of oxidation after the same period of time appears to reinforce the view that the process was not enzymic and hence nonspecific. The uptake of glucose was not inhibited by cyanide, although respiration was completely inhibited for several hours.

Table 2. Estimation of water uptake by tritium labelling in media containing glucose in the presence and absence of ammonium sulphate

Corresponding spore swelling and dry-weight increases are also shown.



The extent of water uptake

The extent of water uptake was estimated comparatively by using tritiated water with samples of conidia shaken in glucose medium, with and without ammonium sulphate. The amount of water taken up was calculated as the excess over a buffer control shaken for the same period of time. The results of this experiment together with dry-weight changes and conidial size changes are given in Table 2 . The results show that the conidia shaken in glucose alone, while showing no increase in width, did show an increase in dry weight and free-water content. The conidia shaken in glucose + ammonium sulphate, however, showed a marked swelling and a larger increase in free water; therefore the swelling may at least in part be attributed to water uptake. Conidia shaken in glucose + ammonium sulphate displayed a $17 \cdot 4 \%$ increase in width over conidia shaken in glucose alone; coupled with this was a $6.2 \%$ increase in dry weight and a $21.1 \%$ increase in water content of the organisms. It is therefore reasonable to say that the main part of the swelling which took place 
in the presence of ammonium sulphate was due to an enhanced capacity to take up water, although the difference in dry weight may also contribute something to the swelling.

\section{DISCUSSION}

The macroconidia of Fusarium culmorum are only able to germinate satisfactorily when supplied with carbon and nitrogen sources. The basal respiration value of the conidia is very low and the necessity for an external carbon source undoubtedly arises from the need for carbon skeletons and high respiratory activity. The way in which a nitrogen source is able to cause the swelling of the conidia by water uptake is not clear. It may be that a nitrogen source, in its role as a wall component, permits the walls to become more elastic, or it may directly affect the permeability properties of the wall. The swelling process, as observed optically, is a roughly linear process and therefore the necessity for a nitrogen source as a precursor for enzyme protein synthesis seems to be eliminated. The stimulation of oxygen uptake during the oxidation of glucose in the presence of a nitrogen source does suggest that the latter is playing a major role in switching the metabolic path of carbon in a germinating conidium. As a limited amount of carbon source is exhausted at the same time in the presence or absence of a nitrogen source it seems that the nitrogen effect is not simply one of rate of utilization.

Ekundayo \& Carlile (1964) suggested that in Rhizopus arrhizus the uptake of water by conidia in the presence of glucose was an active process. In Fusarium culmorum water uptake is only allowed in the presence of a nitrogen source and therefore the process could be termed active in that there is some process other than, or complementary to, osmosis which controls water uptake. Yarwood (1950) suggested that fungal conidia have to attain a degree of hydration comparable to that of powdery mildews before germ-tube emergence can occur. But Ekundayo \& Carlile (1964), in the light of their work, suggested that this hypothesis was untenable, and the results of the present work indicate that some modification is necessary. In view of the several roles of a nitrogen source in germination it seems that the uptake of water may be only subsidiary to other changes in the conidia, which culminate in germination.

The authors thank the Science Research Council for a grant held by R.M. during the completion of this work.

\section{REFERENCES}

Cochrane, J. C., Cochrane, V. W., Simon, F. G. \& Spaeth, J. (1963). Spore germination and carbon metabolism in Fusarium solani. 1. Requirements for spore germination. Phytopathology, 53, 1155.

Cochrane, V. W., Cochrane, J. C., Vogel, J. M. \& Coles, R. S. (1963). Spore germination and carbon metabolism in Fusarium solani. IV. Metabolism of ethanol and acetate. J. Bact. 86, 312.

Exundayo, J. A. \& Carifle, M. J. (1964). The germination of sporangiospores of Rhizopus arrhizus; spore swelling and germ tube emergence. J. gen. Microbiol. 35, 261.

GodDARD, D. R. (1935). The reversible heat activation inducing germination and increased respiration in ascospores of Neurospora tetrasperma. J. gen. Physiol. 19, 45.

HAFIz, A. \& Niederpruem, D. J. (1963). Studies on basidiospore germination in Schizophyllum commune. Am. J. Bot. 50, 614. 
Ratrs, T., Hafiz, A., Nuederpruem, D. J. \& Egbert, P. (1964). Physiological studies on basidiospore germination in Schizophyllum commune. Bact. Proc. p. 111.

SISLER, H. D. \& Cox, C. E. (1954). Effects of tetra-methylthiuram disulfide on metabolism of Fusarium roseum. Am. J. Bot. 41, 338.

Sussman, A. S. (1954). Changes in permeability of ascospores of Neurospora tetrasperma during germination. J. gen. Physiol. 38, 59.

WILson, I. M. (1965). Development of the perithecium and ascospores of Ceriosporopsis halima. Trans. Br. mycol. Soc. 48, 19.

YARWOOD, C. E. (1950). Water content of fungus spores. Am. J. Bot. 37, 636.

\section{EXPLANATION OF PLATE}

Photomicrographs of macroconidia of Fusarium culmorum stained in Sudan Black B to show the spore swelling associated with germination.

Fig. 1. Freshly harvested conidia. $\times 1000$.

Fig. 2. Conidia shaken for $4 \mathrm{hr}$ in a medium containing glucose and ammonium sulphate. $\times 1000$. 

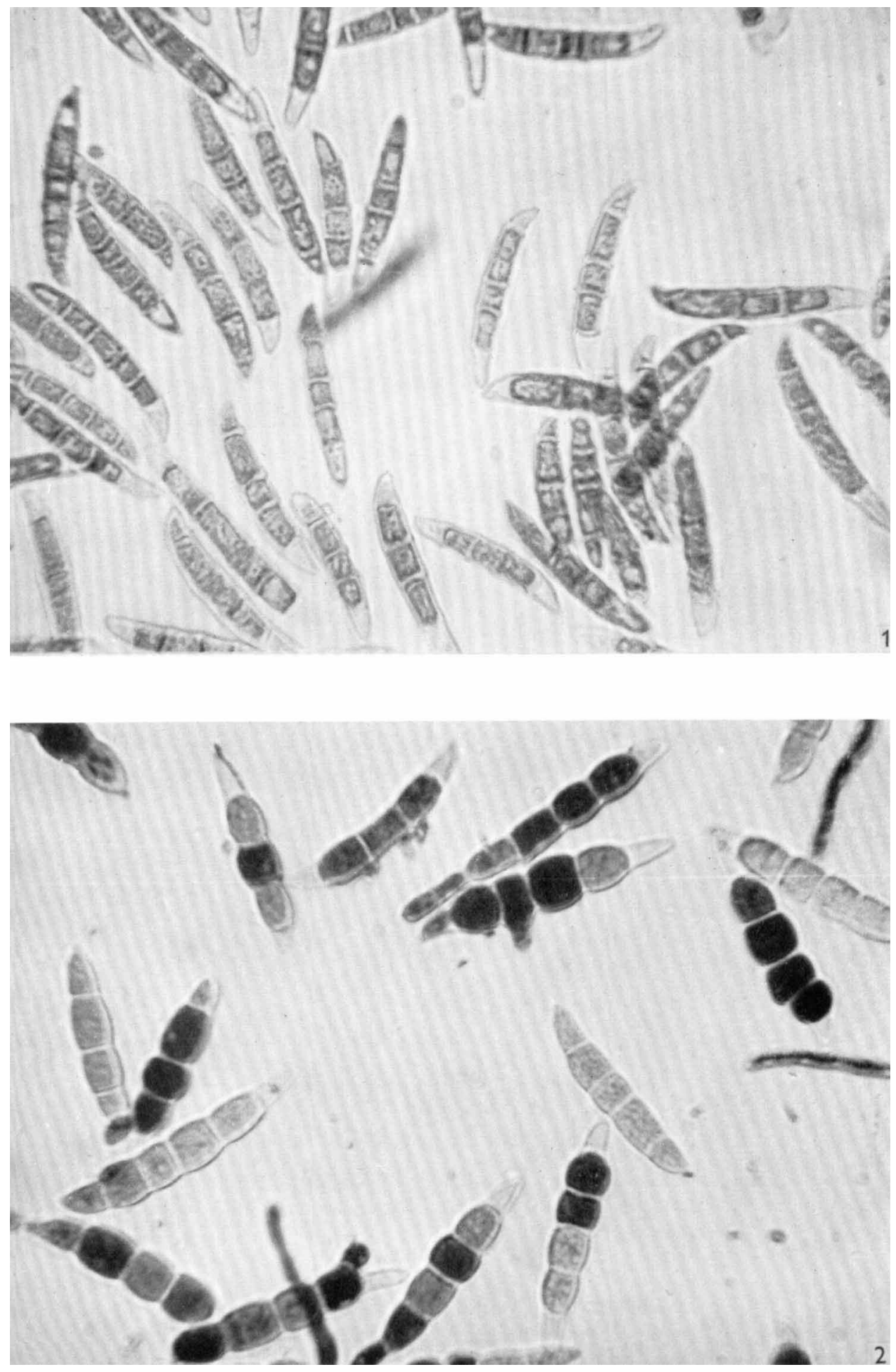